\title{
METALLICITY INHOMOGENEITIES IN LOCAL STAR-FORMING GALAXIES AS A SIGN OF RECENT METAL-POOR GAS ACCRETION
}

\author{
J. SÁnChez Almeida ${ }^{1,2}$, A. B. Morales-Luis ${ }^{1,2}$, C. Muñoz-Tuñón ${ }^{1,2}$, \\ D. M. Elmegreen ${ }^{3}$, B. G. Elmegreen ${ }^{4}$, AND J. MÉNDEZ-Abreu ${ }^{1,2,5}$ \\ ${ }^{1}$ Instituto de Astrofísica de Canarias, E-38205 La Laguna, Tenerife, Spain; jos@iac.es, abml@iac.es, cmt@iac.es \\ 2 Departamento de Astrofísica, Universidad de La Laguna, E-38205 Tenerife, Spain \\ ${ }^{3}$ Department of Physics and Astronomy, Vassar College, Poughkeepsie, NY 12604, USA; elmegreen@ vassar.edu \\ ${ }^{4}$ IBM Research Division, T.J. Watson Research Center, Yorktown Heights, NY 10598, USA; bge@us.ibm.com \\ ${ }^{5}$ School of Physics and Astronomy, University of St Andrews, North Haugh, St Andrews KY16 9SS, UK; jma20@st-andrews.ac.uk \\ Received 2013 October 24; accepted 2014 January 8; published 2014 February 12
}

\begin{abstract}
We measure the oxygen metallicity of the ionized gas along the major axis of seven dwarf star-forming galaxies. Two of them, SDSSJ1647+21 and SDSSJ2238+14, show $\simeq 0.5$ dex metallicity decrements in inner regions with enhanced star formation activity. This behavior is similar to the metallicity drop observed in a number of local tadpole galaxies by Sánchez Almeida et al., and was interpreted as showing early stages of assembling in disk galaxies, with the star formation sustained by external metal-poor gas accretion. The agreement with tadpoles has several implications. (1) It proves that galaxies other than the local tadpoles present the same unusual metallicity pattern. (2) Our metallicity inhomogeneities were inferred using the direct method, thus discarding systematic errors usually attributed to other methods. (3) Taken together with the tadpole data, our findings suggest a threshold around one-tenth the solar value for the metallicity drops to show up. Although galaxies with clear metallicity drops are rare, the physical mechanism responsible for them may sustain a significant part of the star formation activity in the local universe. We argue that the star formation dependence of the mass-metallicity relationship, as well as other general properties followed by most local disk galaxies, is naturally interpreted as side effects of pristine gas infall. Alternatives to the metal-poor gas accretion are examined as well.
\end{abstract}

Key words: galaxies: abundances - galaxies: dwarf - galaxies: evolution - galaxies: formation - galaxies: kinematics and dynamics - galaxies: structure

Online-only material: color figures

\section{INTRODUCTION}

There are two major modes of galaxy formation, as inferred from cosmological numerical simulations (e.g., Silk \& Mamon 2012; Dekel \& Birnboim 2006). At large redshifts major mergers play the dominant role, where galaxies of similar masses merge to form larger aggregates. As the universe evolves, a second mechanism takes over. The protogalaxies grow by accretion of external flows of pristine gas that penetrate the dark matter halo and hit and heat a preexisting elementary disk. Cosmological simulations predict this cold-flow buildup to be the main mode of galaxy formation (Dekel et al. 2009a; Genel et al. 2012), and the incoming gas is expected to form giant clumps that spiral in and merge into a central spheroid (Noguchi 1999; Genzel et al. 2008; Elmegreen et al. 2008) or just to create thick disks that evolve by secular processes (Dekel et al. 2009a; Brook et al. 2012).

Observational evidence for this cold-flow accretion mode comes from the decrease of metallicity associated with internal star formation regions in high-redshift disk galaxies (Cresci et al. 2010). Such localized metallicity drops in the inner disk cannot be explained in any other obvious way except the accretion of external metal-poor gas; secular evolution produces disks with a metallicity decreasing inside out (e.g., Vilchez et al. 1988; van der Kruit \& Freeman 2011; Moran et al. 2012), in sharp contrast to these observations. The same kind of metallicity deficit associated with bright star-forming regions has also been observed in a particular type of local galaxies with tadpole morphology (Sánchez Almeida et al. 2013b). Their images show a large star-forming clump at one end and a long diffuse region to one side. This asymmetric morphology is fairly common at high redshift (Elmegreen et al. 2007; Elmegreen \& Elmegreen 2010; Straughn et al. 2006; Windhorst et al. 2006) but rare in the local universe (Elmegreen et al. 2012b), where it turns out to be associated with extremely metal-poor galaxies and therefore with chemically primitive objects (Papaderos et al. 2008; Morales-Luis et al. 2011; Filho et al. 2013). These facts were used by Elmegreen et al. (2012b) and Sánchez Almeida et al. (2013b) to conjecture that local tadpole galaxies are disks in early stages of assembling. Metallicity drops associated starforming regions have also been observed in a few other local targets, including a gamma-ray burst host galaxy (Levesque et al. 2011) and a blue compact dwarf (BCD) galaxy (Werk et al. 2010). They are interpreted in terms of redistribution of centrally generated metals, with strong galactic winds and subsequent fallback, but not as cold-flow accretion events.

Here we analyze the spatial variation of metallicity in a set of BCD galaxies with intense starbursts that have a range of metallicities from two-thirds to one-twentieth the solar value. The purpose of the work is twofold: first, to see whether the metallicity inhomogeneities observed in tadpoles are also present in other local targets different from the original sample (Section 2) and, second, and equally relevant, to check if the metallicity variations remain when the metallicities are estimated via the direct method. Thus, we can discard a systematic error in the strong-line method employed by Sánchez Almeida et al. (2013b) to infer the abundance inhomogeneities (e.g., Stasińska 2010).

The result of our analysis confirms that, at least in two targets, there are metallicity drops associated with intense starbursts. These drops are not present in the objects of larger metallicity. 
Table 1

Global Parameters of the Galaxies

\begin{tabular}{lcccccccc}
\hline \hline Name $^{\mathrm{a}}$ & $12+\log (\mathrm{O} / \mathrm{H})^{\mathrm{b}}$ & $g^{\mathrm{c}}$ & $\begin{array}{c}\log M_{\star}{ }^{\mathrm{d}} \\
\left(M_{\odot}\right)\end{array}$ & $\begin{array}{c}\mathrm{SFR}^{\mathrm{e}} \\
\left(M_{\odot} \mathrm{yr}^{-1}\right)\end{array}$ & $\begin{array}{c}M_{\star} / \mathrm{SFR}^{\mathrm{f}} \\
(\mathrm{Gyr})\end{array}$ & $\begin{array}{c}\text { Redshift } \\
\times 10^{2}\end{array}$ & Morphology & $\Delta \log (\mathrm{O} / \mathrm{H}) ?$ \\
\hline SDSSJ083713.13+360350.4 & $8.54 \pm 0.01$ & 17.4 & 9.14 & 0.68 & 2.0 & 3.31 & Single knot & No \\
SDSSJ094254.27+340411.8 & $7.79 \pm 0.05$ & 19.1 & 7.34 & 0.33 & 0.068 & 2.25 & Single knot & Unclear \\
SDSSJ100348.65+450457.7 & $7.89 \pm 0.01$ & 17.6 & 7.60 & 0.15 & 0.27 & 0.92 & Single knot & No \\
SDSSJ150934.17+373146.1 & $7.80 \pm 0.01$ & 17.3 & 7.51 & 3.62 & 0.009 & 3.25 & Cometary & No \\
SDSSJ164710.66+210514.5 & $8.11 \pm 0.03$ & 16.9 & 7.73 & 0.43 & 0.12 & 0.91 & Cometary & Yes \\
SDSSJ223831.12+140029.7 & $7.43 \pm 0.01$ & 18.7 & 7.55 & 0.51 & 0.070 & 2.06 & Two-knots & Yes \\
SDSSJ230210.00+004938.8 & $7.75 \pm 0.03$ & 18.8 & 7.27 & 0.75 & 0.025 & 3.31 & Two-knots & Unclear \\
\hline
\end{tabular}

Notes.

${ }^{\text {a }}$ Named so that right ascension and declination are implicit.

${ }^{\mathrm{b}}$ From the spatially integrated spectrum.

${ }^{\mathrm{c}}$ Integrated $g$ magnitude provided by SDSS/DR9.

${ }^{\mathrm{d}}$ Masses from SDSS/DR9 photometry using mass-to-light ratios by Bell \& de Jong (2001).

${ }^{\mathrm{e}} \mathrm{Star}$ formation rate from $\mathrm{H} \alpha$ flux using the prescription in Elmegreen et al. (2012b).

${ }^{\mathrm{f}}$ Time to form all stars in the galaxy at the current SFR: inverse specific SFR.

We use this fact to conjecture that a minimum metallicity around one-tenth the solar value is needed for the metallicity decrements to be observed. Galaxies with metallicities below this one-tenth threshold are usually referred to as extremely metal-poor (XMP; e.g., Kunth \& Östlin 2000).

One might interpret the rarity of galaxies with metallicity drops as evidence against systematic cold-flow accretion in the local universe. Thus, the few observed decrements would represent vestiges of a physical process common early on but now almost inoperative. However, several independent observations suggest that star formation triggered by accretion of metal-poor (perhaps pristine) gas may be a process more common than anticipated. Several of those observations are put forward and discussed in detail in Section 5, all of them involving global properties of large numbers of galaxies. The most conspicuous one refers to the so-called mass-metallicity relation. It has recently been found (Mannucci et al. 2010; Lara-López et al. 2010; Pérez-Montero et al. 2013; Andrews \& Martini 2013) that for galaxies of the same mass, the current star formation rate (SFR) is anticorrelated with the ionized gas metallicity. No contrived explanation is required if the two parameters are physically connected, as if the infall of metalpoor gas feeds and triggers the star formation in these galaxies (Brisbin \& Harwit 2012; Davé et al. 2012).

The paper is organized as follows. Section 2 describes observations and reduction. Metallicity estimates are outlined in Section 2.1. The resulting gradients and inhomogeneities are analyzed in Section 3. Potential observational biases and alternatives to the metal-poor gas accretion are examined in Section 4. Observational evidence for grand-scale gas inflows triggering star formation in the local universe is presented and discussed in Section 5. The implications of our work are considered in Section 6.

\section{OBSERVATION AND DATA ANALYSIS}

The seven galaxies used in this study are listed in Table 1. Even though their spectra were originally obtained with a different purpose,${ }^{6}$ they turned out to be ideal for our work. Their

\footnotetext{
6 Specifically, for checking the metallicity of XMP candidates selected from SDSS/DR6 as BCDs having negligible [N II] $\lambda 6583$ (Section 2.2 in Sánchez Almeida et al. 2008). The absence of this line is a signature of low metallicity (e.g., Denicoló et al. 2002; Morales-Luis et al. 2011), but most of the candidates from Sánchez Almeida et al. (2008) lack [N II] $\lambda 6583$ because of an artifact of the reduction pipeline that removed [N II] together with an overlapping telluric line. Thus, they present a range of metallicities.
}

long-slit spectra provide spatial resolution within the targets, with enough spectral coverage to detect all the lines required for oxygen abundance analysis using the direct method. The targets cover a wide range of metallicities, from twothirds to one-twentieth the solar metallicity (see Table 1, with $12+\log (\mathrm{O} / \mathrm{H})_{\odot}=8.69$ as measured by Asplund et al. 2009). In addition, the galaxies form stars actively, in the sense that the current starburst is much larger than the average SFR during the galaxy lifetime (assumed to be similar to the age of universe $t_{0}$ since the galaxies presumedly contain old stellar populations; see, e.g., Papaderos et al. 1996; Corbin et al. 2006; Sánchez Almeida et al. 2012). The timescale $t_{\star}$ to produce their stellar masses $M_{\star}$ at the current SFR,

$$
t_{\star}=M_{\star} / \mathrm{SFR},
$$

is typically much smaller than $1 \mathrm{Gyr}$ (see Table 1) and therefore much smaller than $t_{0}(\simeq 14 \mathrm{Gyr})$. The SFRs and stellar masses in Table 1 use Sloan Digital Sky Survey (SDSS) H $\alpha$ fluxes, colors, and magnitudes together with the prescriptions in Kennicutt (1998) and Elmegreen et al. (2012b) and the mass-to-light ratios in Bell \& de Jong (2001).

All long-slit spectra were taken with the ISIS spectrograph of the $4.2 \mathrm{~m}$ William Hershel Telescope (WHT) operated in the Roque de los Muchachos Observatory. ${ }^{7}$ The dual beam (red and blue) covers in a single exposure from $\lambda 3600$ to $8000 \AA$. The ISIS@WHT setup includes intermediate gratings that after a $2 \times 2$ binning of the CCD, provide $1.7 \AA$ pixel $^{-1}$ (blue) and $1.9 \AA_{\text { pixel }}^{-1}$ (red) equivalent to 0.40 pixel $^{-1}$ (blue) and 0.44 pixel $^{-1}$ (red). We use a slit $1^{\prime \prime}$ wide, which limits the angular resolution and also sets the spectral resolution to $4.2 \AA$ in both the red and blue arms. This resolution suffices to measure the fluxes of the relevant emission lines [O III] $\lambda \lambda 4363,4959$, $5007 \AA,\left[\mathrm{O}_{\mathrm{II}}\right] \lambda \lambda 3727,7319,7330 \AA, \mathrm{H} \beta, \mathrm{H} \alpha,[\mathrm{N}$ II $] \lambda 6584$, and [S II] $\lambda \lambda 6717,6731 \AA$. The observations were carried out in two campaigns (2009 January 31 and 2010 July 15), both with fair to good seeing conditions from 1".3 and 0.'5. We integrated $4000 \mathrm{~s}$ on target. Some of the objects show an elongated morphology (Table 1), and then the slit was oriented along the major axis. Otherwise, the slit followed the parallactic angle.

The reduction procedure included standard bias and flat-field corrections, cosmic ray elimination, absolute flux calibration,

\footnotetext{
7 http://www.ing.iac.es/astronomy/telescopes/wht/
} 

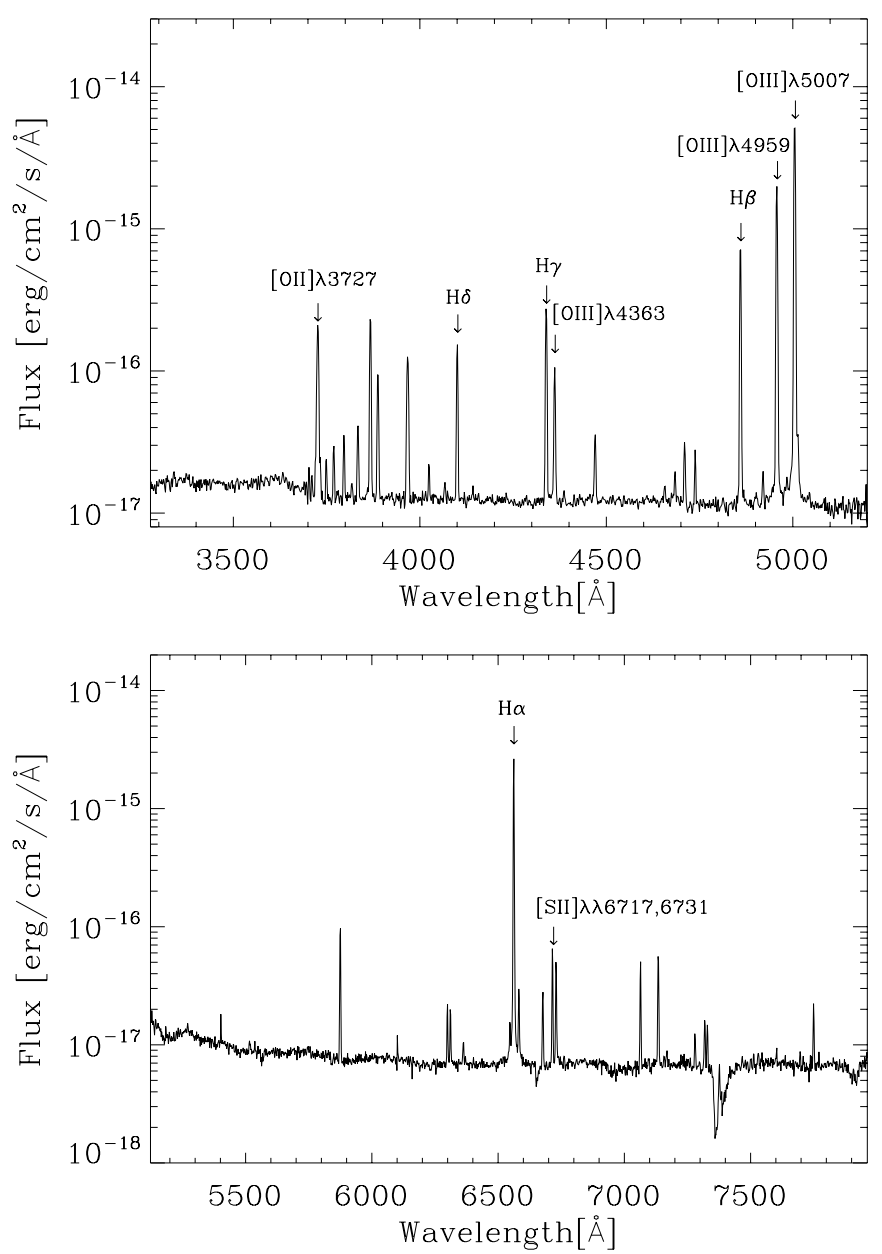

Figure 1. Example of one of the spectra used in this work, with the main emission lines included. It corresponds to the brightest knot of J1509+37. The spectra of the two arms of the spectrograph are shown in different panels; the top and bottom panels correspond to the blue and red arms, respectively. Fluxes are given in a logarithmic scale to show faint lines.

and removal of sky emission lines. Spectral and spatial directions were not exactly perpendicular on the CCD, and we also correct for this effect. The spectra were aligned so that each column corresponds to a single position in the sky. Thus, the different columns are extracted and analyzed independently in this paper, with each spectrum representing 0. .44 of the galaxy. After these manipulations, the signal-to-noise ratio $(\mathrm{S} / \mathrm{N})$ in $\mathrm{H} \alpha$ turns out to be between 1000 and 300 from the center to the outskirts of a typical galaxy. The critical line needed for electron temperature determination, $[\mathrm{O} \mathrm{III}] \lambda 4363$, is much fainter than $\mathrm{H} \alpha$, but it still reaches a $\mathrm{S} / \mathrm{N}$ up to 70 in the brighter regions. As we explain below, $\mathrm{S} / \mathrm{N} \rightarrow 0$ (this line disappears) when the metallicity becomes large. Figure 1 contains an example of one of these fully reduced spectra, specifically, the brightest knot of J1509+37 (Table 1).

\subsection{Metallicity Determination}

We determine the oxygen abundance using the direct method (e.g., Shaw \& Dufour 1995; Stasińska 2004), following the prescription by Hägele et al. (2008), which includes employing the Balmer decrement to correct for internal reddening. Electron densities were calculated using the ratio of fluxes [S II] $\lambda 6717 /$ [S II] $\lambda 6731$. The electron temperature of [O III] was derived from the ratio $([\mathrm{O}$ III $] \lambda 4959+[\mathrm{O}$ III $] \lambda 5007) /[\mathrm{O}$ III $] \lambda 4363$. The ratio [O II $] \lambda 3727 /([\mathrm{O} \mathrm{II}] \lambda 7319+[\mathrm{O} \mathrm{II}] \lambda 7330)$ was used to measure the electron temperature of [O II]; when this line was not available, it was calculated using the relationship between [O II] and [O III] temperatures worked out by Pérez-Montero \& Díaz (2003). Throughout this paper we use the term modified direct method to describe this approach to the [O II] temperature estimate. Finally, the oxygen metallicity is computed by adding up the contribution of all oxygen ionization states up to $\mathrm{O}^{2+}$. The errors in the oxygen abundances were computed in a Monte Carlo simulation by randomly modifying the fluxes of the emission lines according to the noise of the observed spectra as measured in their continua and scaled up to account for the photon noise (e.g., Pérez-Montero \& Díaz 2003, Section 3.1). The abundances are computed from 500 realizations of the noise, and the standard deviation of the resulting $\mathrm{O} / \mathrm{H}$ are quoted as error bars. In a second error estimate, we repeated the Monte Carlo exercise assuming the noise in the continuum was three times the observed one.

In addition to the direct method, in order to compare it with the metallicities and metallicity variations found by Sánchez Almeida et al. (2013b), we also estimate the oxygen abundance using the ratio [N $\mathrm{NI}] \lambda 6583$ to $\mathrm{H} \alpha$. It is the so-called N2 method as proposed by Denicoló et al. (2002), and we use it in the calibration by Pérez-Montero \& Contini (2009).

\section{SPATIAL VARIATION OF METALLICITY}

The direct-method-based oxygen abundance corresponding to the spatially integrated spectra of all the observed galaxies is given in Table 1. It represents the luminosity-weighted average metallicity. Even though all targets are metal-poor, only $\mathbf{J} 2238+14$ is XMP in the usual sense of having an average metallicity smaller than a tenth of the solar value (i.e., $12+\log (\mathrm{O} / \mathrm{H}) \leqslant 7.69 ;$ Asplund et al. 2009, and Section 1).

Figures 2-4 show the three types of observed spatial variations. The SDSS (Stoughton et al. 2002; Ahn et al. 2012) images on top indicate the orientation of the slit. The bottom panels plot oxygen abundance versus position along the slit in arcseconds, using as a reference position the pixel of the largest $\mathrm{H} \alpha$ flux. Abundances inferred from the direct method are represented as black dots joined by black solid lines. These are the measurements we discuss unless otherwise stated. Abundances from the modified direct method (blue lines) and N2 method (red lines) are analyzed later on. The targets J0942+34 and $\mathrm{J} 2302+00$ behave similarly (the latter represented in Figure 2) in the sense that the spatial region with enough $\mathrm{S} / \mathrm{N}$ to carry out the metallicity measurement is too small to provide any reliable spatial variation. Seeing during observation was of the order of $1^{\prime \prime}$ (Section 2), which is similar to the spatial extent of the signals on the CCDs (see the continuum and $\mathrm{H} \alpha$ fluxes in Figure 2, represented as the orange and green histograms, respectively). Figure 3 (black solid line) shows a rather constant metallicity, and this time the galaxy is significantly larger than the seeing. Figure 3 displays J1509+37, but its behavior also stands for $\mathrm{J} 1003+45$. These galaxies show no obvious metallicity gradient or drop. Finally, Figure 4 portrays $\mathrm{J} 2238+14$, which clearly shows two metallicity decrements associated with the two bright knots of the galaxy (compare the black solid line representing $\mathrm{O} / \mathrm{H}$ with the $\mathrm{H} \alpha$ and continuum fluxes shown as histograms). The metallicity drop corresponds to $\Delta[12+\log (\mathrm{O} / \mathrm{H})] \simeq-0.5 . \mathrm{J} 2238+14$ is the galaxy with the lowest average metallicity in the sample (see the dashed line corresponding to $\mathrm{O} / \mathrm{H}=(\mathrm{O} / \mathrm{H})_{\odot} / 10$, which is common to Figures 2-4). Figure 5 is similar to Figure 4 in the sense that it shows a significant spatial variation of metallicity for J1647+21. 

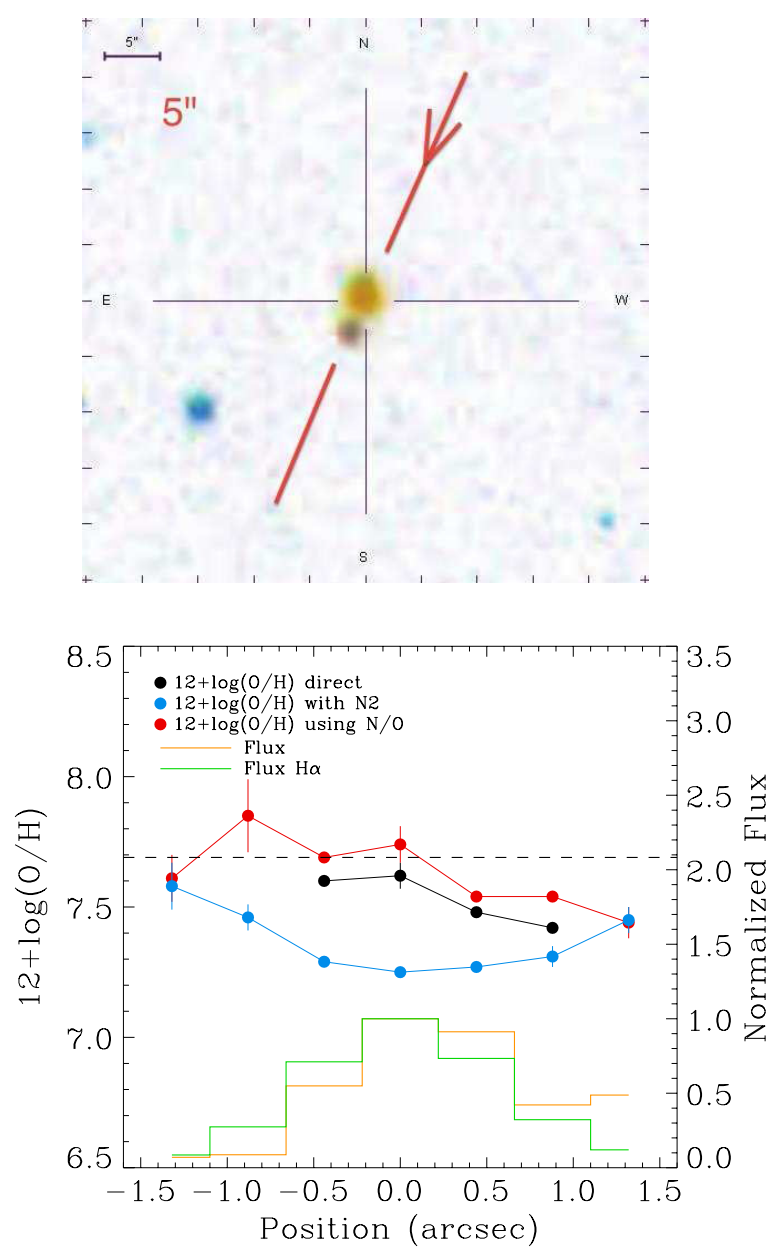

Figure 2. Top: color-code inverted SDSS image of galaxy J2302+00. The red line indicates the orientation of the spectrograph slit, with the arrow pointing in the sense of growing position along the slit. The scale corresponds to $5^{\prime \prime}$ on the sky. Bottom: metallicity and flux variation along the slit of this target. We show the metallicity computed using the direct method (black solid line joining black points), the modified direct method described in Section 2.1 (red solid line), and the N2 method (blue solid line). The flux of the integrated spectrum and the $\mathrm{H} \alpha$ flux are given as orange and green histograms, respectively. Their values have been normalized to the largest flux, and the ordinate axis on the right-hand side of the plot refers to them. The scale of oxygen metallicity is given on the left-hand side of the plot. Positions along the slit are in arcseconds referring to the point of the largest $\mathrm{H} \alpha$ flux. In this particular target, only the main galaxy knot is detected. The extent is too small compared to seeing to decide whether there are significant variations of metallicity. The dashed line indicates a tenth of the solar metallicity and is used for reference. The error bars account only for random noise in the observed spectra. Other sources of error are included in Figure 3.

(A color version of this figure is available in the online journal.)

The source is larger and more complex than J2238+14 (Figure 5). The long slit spectrum does not have enough signal for metallicity analysis in between the two main galaxy knots (except for a single pixel in between; see Figure 5). However, the signals in the knots clearly indicate a significant difference of metallicity. The brightest one (at position zero) has a metallicity of the order of one-tenth of the solar value, whereas the second one (at positions between $-35^{\prime \prime}$ and $-40^{\prime \prime}$ ) is doubtless more metal-rich, even though we cannot assess its actual metallicity. The spectrum in the metal-rich knot does not show [O III] $\lambda 4363$, which is needed for electron temperature estimates. However, this lack implies a low electron temperature and therefore a high metallicity (McGaugh 1991; Sánchez Almeida et al. 2012). We estimate a lower limit of $12+\log (\mathrm{O} / \mathrm{H}) \geqslant 8.2$, computing the metallicity with an [O III] $\lambda 4363$ flux just below the continuum
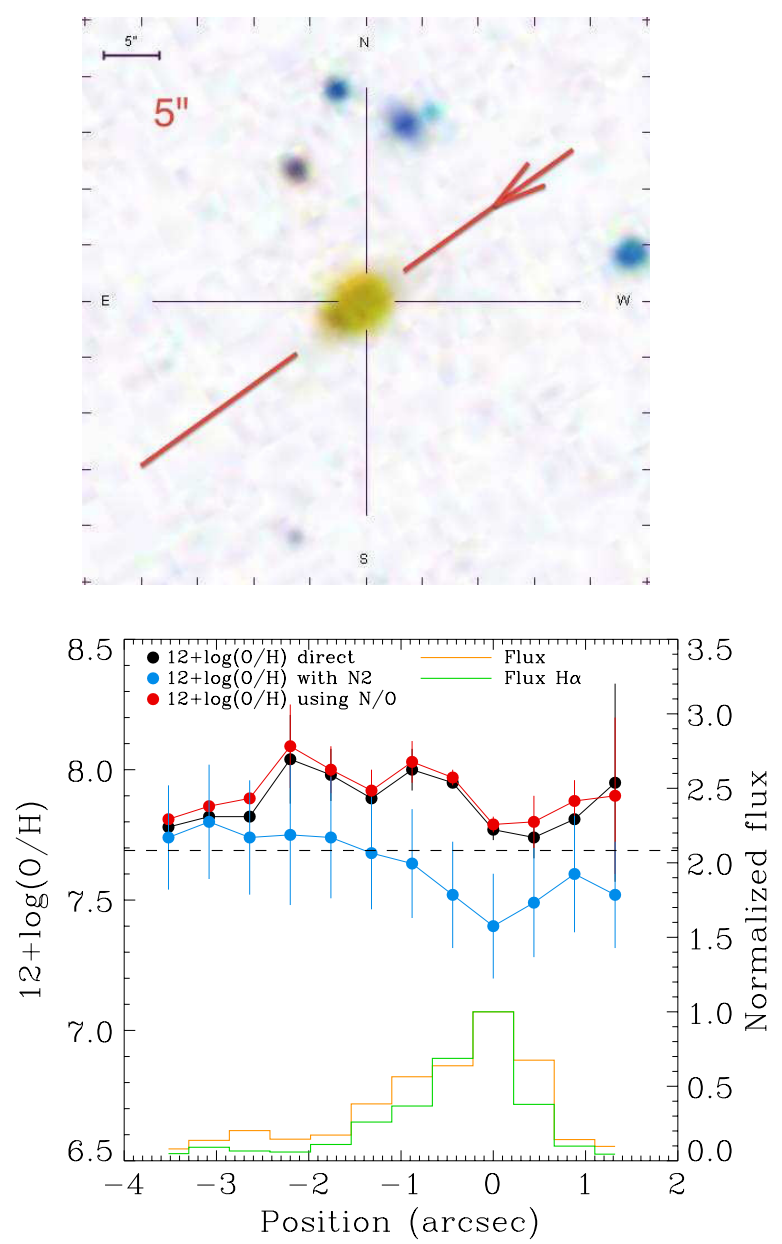

Figure 3. Same as Figure 2, but corresponding to the target J1509+37. This time the spectral signals extend over a region larger than the seeing, so we detect no obvious metallicity variation along the slit. For the meaning of the various axes, curves, and symbols, see Figure 2. The dashed line corresponds to $1 / 10$ of the solar metallicity. The continuum noise has been artificially increased by a factor of three to compute the error bars in this plot. In addition, the N2 abundance errors have been enlarged by 0.2 dex to include the scatter of the $\mathrm{N} 2$ metallicity calibration.

(A color version of this figure is available in the online journal.)

noise level in our spectra. This lower limit is represented in Figure 5.

Table 1 contains a flag indicating whether the metallicity variations are present in the galaxies, are not present, or are unclear. It is unclear in the two galaxies that are too small. Discarding them, $40 \%$ of the galaxies show metallicity drops (two out of five objects).

Figures 2-5 include metallicities derived from the semiempirical N2 method (blue dots joined by blue solid lines). Overall, they show the same trends and drops as the direct method, confirming that at least for these targets both techniques provide qualitatively consistent results. In some cases there are small differences, e.g., the drop of N2 metallicity in J1509+37, which is not obvious in the direct-method-based metallicity (Figure 3). However, these discrepancies are within the 0.2 dex scatter typical of the N2 calibration (e.g., Pettini \& Pagel 2004; PérezMontero \& Contini 2009). This is more clear in Figure 3, where the error bars include both the noise in the spectra plus $0.2 \mathrm{dex}$ ascribed to the N2 calibration. Figures 2-5 also include oxygen abundances inferred from the alternative direct method (Section 2.1), and they also agree with the rest (see the red solid lines). The error bars propagated from the noise in the 

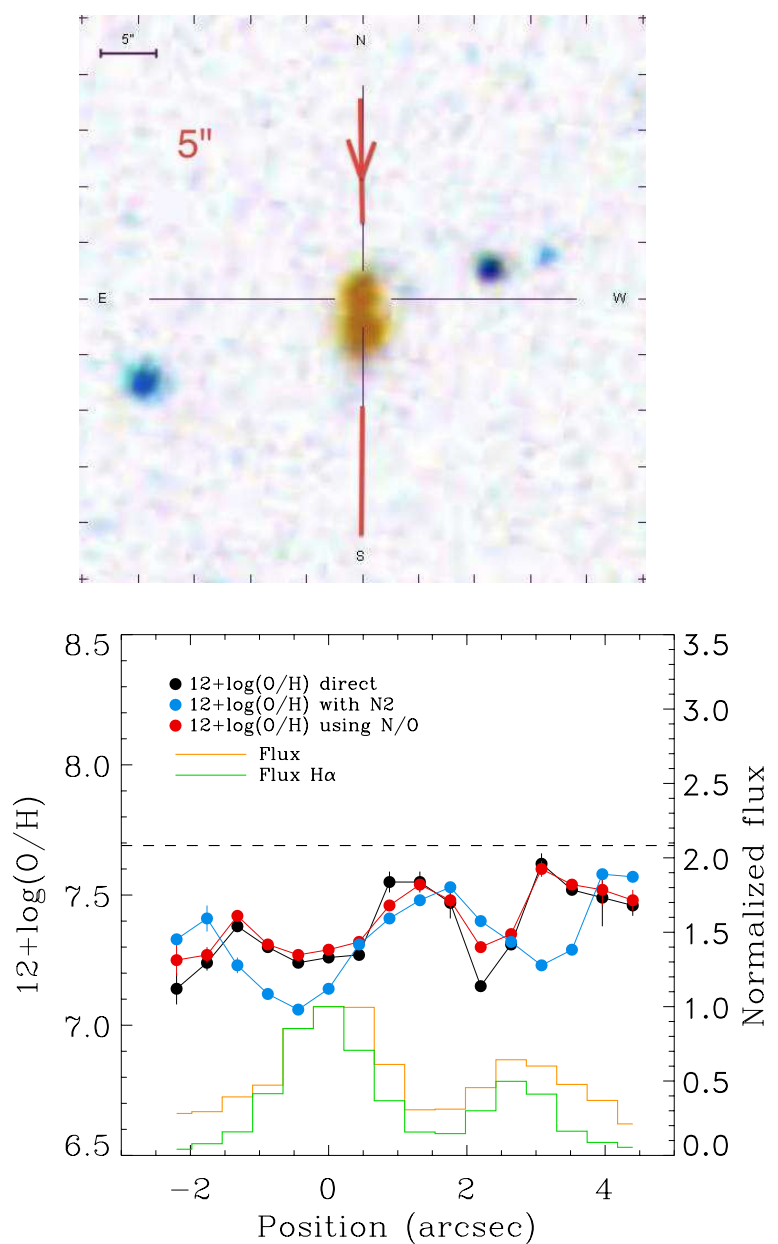

Figure 4. Same as Figure 2, but corresponding to J2238+14. The target is larger than the seeing, and it shows a clear metallicity variation along the slit (black solid line) with a pattern similar to the $\mathrm{H} \alpha$ flux variation (green histogram). For the meaning of the other curves and symbols, see Figure 2. The dashed line corresponds to one-tenth of the solar metallicity. The error bars account only for random noise in the observed spectra. Other sources of error are included in Figure 3.

(A color version of this figure is available in the online journal.)

spectra (Section 2.1) are unrealistically small for reasons that we ascribed to bias in the flux estimates that were not accounted for when propagating the continuum error. In order to make them more realistic, errors were also computed by increasing the observed continuum noise by a factor of three. These larger error bars are only represented in Figure 3.

Galaxy J0837+36 has not been mentioned so far because its case slightly differs from the rest. It is more massive and has a lower specific SFR (i.e., SFR/ $M_{\star}$; Table 1). Its metallicity is so large that [O III] $\lambda 4363$ is not detectable in individual spatial pixels, so direct method metallicity gradients cannot be computed. As we explained above for the high-metallicity knot of J1647+21, the absence of this line proves the high metallicity of the H II gas, even though we cannot quantify it. From the N2-based estimate, we conclude that the metallicity variations of this galaxy are negligibly small, as indicated in the last column of Table 1.

\section{ALTERNATIVES TO THE INFERRED METALLICITY DECREMENTS}

This section analyzes alternatives to explaining the observed metallicity drops as the outcome of a metal-poor gas accretion
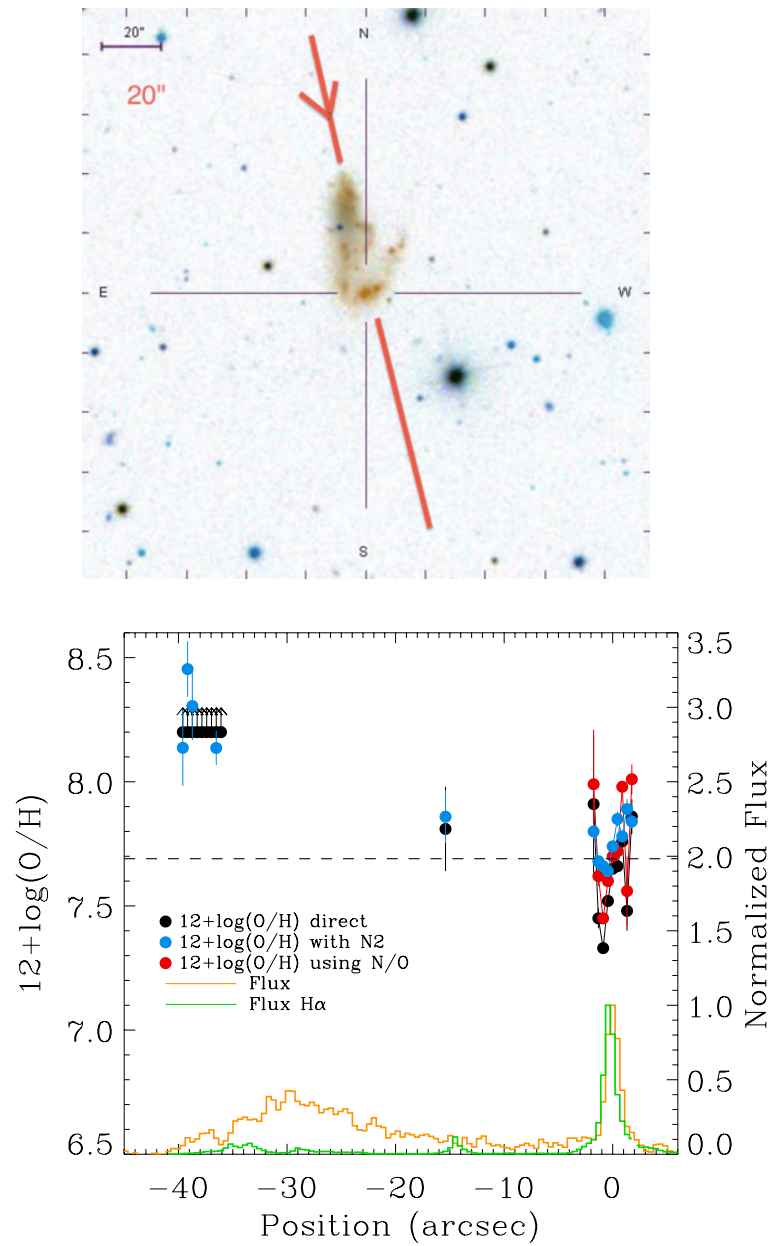

Figure 5. Same as Figure 2, but corresponding to J1647+21. The galaxy is larger than the seeing, and it shows metallicity variations along the slit (black dots), with a pattern similar to the $\mathrm{H} \alpha$ flux variation (green histograms). For the meaning of the other curves and symbols, see Figure 2. The zero of the position scale in the bottom plot corresponds to the center of the large cross in the top image. Positions along the slit grow in the sense indicated by the arrow on the top image. The error bars account only for random noise in the observed spectra. Other sources of error are included in Figure 3.

(A color version of this figure is available in the online journal.)

event. Specifically, we point out possible biases of the direct method that artificially produce low metallicities (Section 2.1), as well as a mechanism that may lead to the metal impoverishment of regions with long-lasting intense starbursts. Even though these potential problems cannot be fully ruled out, accretion of pristine gas remains the simplest way of explaining the observations.

If the temperature in the region is not homogeneous, then the direct method underestimates the true abundances (Peimbert 1967; Peimbert \& Costero 1969). There is a longlasting debate in the literature on whether such temperature fluctuation exists (Stasińska et al. 2013 and references therein). An artificial reduction of metallicity of 0.5 dex can be produced by temperature fluctuations of the order of $20 \%-30 \%$ rms (e.g., Stasińska 2004; Esteban et al. 2009). If this effect is responsible for the observed drop of metallicity, then such fluctuations should be localized in the low-metallicity starburst but not in the rest of the galaxy. We cannot rule out this possibility since the interstellar medium (ISM) of our targets is poorly known. We note, however, that the physical mechanisms proposed to generate temperature inhomogeneities favor high-metallicity media 
rather than our XMP galaxies (see the review by Torres-Peimbert $\&$ Peimbert 2003). For example, they require dust particles for heating (e.g., Stasińska \& Szczerba 2001), or they need metals for the metallicity inhomogeneities to cause the temperature fluctuations (e.g., Kingdon \& Ferland 1998).

The direct method does not consider the presence of density fluctuations, which in some real cases may be large. The resulting electron density variations are not expected to have significant impact on the abundances (e.g., Stasińska et al. 2012); however, density inhomogeneities may have an indirect influence through induced temperature inhomogeneities (e.g., Torres-Peimbert \& Peimbert 2003). If the plasma is heated by collisions with photoionized electrons, dense clumps present lower temperatures, leading to temperature fluctuations. Detailed tailored modeling is required to assess the practical importance of the effect. If it is meant to explain the observed metallicity drops, the largest density fluctuations must occur where the metallicity appears to be lowest.

In order to determine the oxygen abundance, the standard direct method used in this paper includes only $\mathrm{O}, \mathrm{O}^{+}$, and $\mathrm{O}^{2+}$ but not higher ionization states. Since the ionizing radiation field is harder in young star-forming regions, one may wonder whether our metallicity drops are actually caused by overlooking $\mathrm{O}^{3+}$ in large starbursts. However, this potential bias does not explain the magnitude of the observed drops. Even when very hot stars are present in $\mathrm{H}$ II regions, the correction for unseen states of oxygen is negligibly small with respect to other sources of errors (e.g., Stasińska et al. 2012). The ionization correction factors for $\mathrm{O}^{3+}$ are never of the order of $0.5 \mathrm{dex}$, as required to reproduce our observations (e.g., Kingsburgh \& Barlow 1994).

Dwarf galaxies have shallow gravitational potentials that cannot retain all the metals ejected by supernova (SN) explosions (e.g., Mac Low \& Ferrara 1999). Therefore, their metal enrichment depends critically on two competing processes, both controlled by the SN rate, that is to say, controlled by the SFR: the metal production and the metal loss. Both increase with the SFR. Because of this interplay, dwarfs may enrich more efficiently at mild SFRs, where the two opposite effects reach a compromise (Hidalgo et al. 2011; Koleva et al. 2013). This trade-off between SFR and metallicity may induce the metallicity pattern that we observe. If a major starburst has been losing most of the metals for long, the gas around it would have a metallicity lower than the rest of the galaxy, where the star formation has proceeded at a lower, more efficient rate. Even though we cannot fully discard this possibility, we envisage two difficulties for this explanation to work with our targets. First, the high star formation mode quickly exhausts the original gas supply, which has to be replenished with metal-poor gas that does not exist in the galaxy. Second, and equally important, the gas in the starbursting region should remain unmixed, or the full galaxy would acquire a uniform metallicity. This is not easy to attain since mixing mechanisms are expected to efficiently operate on short timescales (megayears; e.g., Tenorio-Tagle 1996; de Avillez \& Mac Low 2002).

\section{UBIQUITY OF THE PHENOMENON}

A number of observational properties characterizing large samples of star-forming galaxies can be naturally explained if the metallicity drop associated with intense starbursts is a common phenomenon. The inflow of pristine gas provides a simple physical unifying mechanism that explains all of them, even though often it is not the only explanation of each individual observation. This section critically reviews some of these results in terms of metal-poor gas inflow triggering star formation. The discussions are fairly qualitative, emphasizing the diversity of observations hinting at grand-scale metal-poor gas inflows in the local galaxies.

\subsection{Mass-Metallicity-Star Formation Rate Relationship}

Local galaxies are known to follow a mass-metallicity relationship, where the larger the mass is, the higher the metallicity is (e.g., Skillman et al. 1989; Tremonti et al. 2004; Gallazzi et al. 2005). The relationship presents a significant scatter that has recently been found to be associated with the present SFR in the galaxy ${ }^{8}$ (Mannucci et al. 2010; Lara-López et al. 2010; Yates et al. 2012; Pérez-Montero et al. 2013; Andrews \& Martini 2013; Zahid et al. 2013). Specifically, for galaxies with the same stellar mass, the metallicity decreases as the current SFR increases. The mass-metallicity relationship is commonly interpreted as being due to variations of the star formation efficiency with galaxy mass and/or to galaxy massdependent metal-rich outflows (e.g., Lee et al. 2008; Ellison et al. 2008). The former implies that low-mass galaxies produce fewer stars for their gas and so become more metal-poor, whereas the latter relies on the metal-rich SN ejecta to be preferentially lost to the intergalactic medium by low-mass galaxies because of their shallower gravitational well. Neither of these two mechanisms, however, predicts the observed dependence on the SFR of the metallicity: they render a metallicity set only by the galaxy mass. ${ }^{9}$ Conversely, the observed anticorrelation between metallicity and SFR can be qualitatively understood if the star formation is preferentially triggered and sustained by the inflow of metal-poor gas, which has no time to be well mixed with the high-metallicity gas already present in the ISM of the galaxies. The agreement is more than qualitative according to Brisbin \& Harwit (2012). Using a toy model for the gas inflow, these authors conclude that most of the starforming galaxies with stellar masses $M_{\star} \leqslant 2 \times 10^{10} M_{\odot}$ and many with $M_{\star} \geqslant 2 \times 10^{10} M_{\odot}$ appear to be fed by lowmetallicity gas infall. The importance of metal-poor gas infall to account for the observed mass-metallicity-SFR relationship is also emphasized by Davé et al. (2012) and Dayal et al. (2013) in their simple analytic chemical evolution models that include mass infall and outflows. In particular, Davé et al. (2012) explain the metallicity-SFR relationship as transient departures from the secular evolution of the galaxies, triggered by sudden infalls of metal-poor gas.

Unlike the metallicity, the ratio between the observed $\mathrm{N}$ and O does not seem to depend on SFR (see Pérez-Montero et al. 2013; Andrews \& Martini 2013). This lack of SFR dependence is consistent with the relation between metallicity and SFR being maintained by episodic metal-poor inflows. The advent of fresh gas triggers star formation and drops the metallicity, but it does not change the preexisting relative abundance between metals.

The mass-metallicity-SFR relationship is followed by large numbers of star-forming galaxies, so it represents a behavior common to the typical galaxies of the local universe. It is not restricted to a few rare vestigial objects. Therefore, if the above conjecture turns out to be correct and pristine gas

\footnotetext{
8 Often referred to as the fundamental mass-metallicity relationship (Mannucci et al. 2010).

9 At least in simple chemical evolution models. Even if the outflow rate scales with the SFR, the metallicity of the gas is set by the gas fraction, but this gas fraction depends only on the stellar mass (e.g., Edmunds 1990). Thus, given the stellar mass, the metallicity is fixed, leaving no room for a SFR-metallicity correlation.
} 
infall is responsible for the SFR dependence, then this infall is a characteristic of the full population of local star-forming galaxies. This conclusion is the central point of the section.

\subsection{High Metallicity of Quiescent BCDs}

BCD galaxies are high-surface-brightness targets and thus are relatively easy to detect. Most XMPs are also BCDs (e.g., Kunth $\&$ Östlin 2000; Morales-Luis et al. 2011). The luminosity of these galaxies is dominated by one or several young starbursts; however, most, if not all, BCDs contain host galaxies with old stars (e.g., Papaderos et al. 1996; Cairós et al. 2003; Corbin et al. 2006; Amorín et al. 2007). The dominant starburst is so intense that it cannot be sustained for long; therefore, the BCDs have to be in a transient phase. (Using the arguments and the symbols in Section 2, their $t_{\star} \ll t_{0}$.) Consequently, there must be many local galaxies in the pre- or post-BCD phase, i.e., many quiescent BCDs (or, for short, QBCDs).

The BCD hosts show up in the galaxy outskirts; therefore, deep photometry allowed Amorín et al. $(2007,2009)$ to characterize their photometric properties. Using the typical host colors and magnitudes as proxies for QBCD properties, Sánchez Almeida et al. (2008) searched the SDSS/DR6 archive for QBCD candidates. They turned out to be rather common: 1 out of 3 local dwarf galaxies is of this kind, and there are some 30 of them per BCD galaxy. Their main properties, including their luminosity functions, are consistent with the BCDs being QBCDs observed during a starburst phase in a duty cycle where the QBCD phase lasts 30 times longer than the BCD phase. This interpretation presents a difficulty, however: the gas phase metallicity of the QBCDs is systematically higher than the metallicity of the BCDs. This cannot happen in a closed-box evolution, where the precursor galaxy always has lower metallicity than the follower, so that QBCDs could not be precursors of BCDs. The problem naturally disappears if almost every BCD starburst is preceded by the advent of fresh metal-poor gas that triggers the star formation episode. Moreover, such a gas infall hypothesis beautifully explains why the stellar metallicities of BCDs and QBCDs agree, even though their gas phase metallicities do not (Sánchez Almeida et al. 2009). The stars of BCDs and QBCDs are statistically the same because only a small fraction of galaxy stellar mass is produced in each starburst. ${ }^{10}$ Their gas differs because BCDs have just rejuvenated their ISM.

This behavior affects not just a few objects, but $30 \%$ of all local dwarfs. Therefore, the gas infall must be a common phenomenon if it is responsible for the metallicity discrepancy between BCDs and QBCDs.

\subsection{Morphology-Metallicity Relationship}

XMP galaxies tend to have cometary or other nonsymmetric morphologies (Papaderos et al. 2008; Morales-Luis et al. 2011; Filho et al. 2013). Even if surprising, such an association seems to be the extreme case of a common relationship between morphology and metallicity followed by the bulk of the starforming galaxies in the local universe. Reichard et al. (2009) parameterize lopsidedness in a sample of $\simeq 2.5 \times 10^{4}$ nearby galaxies and find that at fixed mass, the more metal-poor galaxies are more lopsided. Whatever process causes lopsidedness, it is also associated with low-metallicity gas in the galaxies. In the case of the XMP, the lopsidedness is produced by off-center

\footnotetext{
10 Although in some extreme cases the present burst may be producing a significant fraction of the stellar mass, e.g., our $\mathrm{J} 1509+37$, this is not the general behavior.
}

large $\mathrm{H}$ II regions, fed by pristine gas accretion either directly or indirectly: directly if the gas arrives to the disk ready to form stars (e.g., Dekel et al. 2009a, 2009b) or indirectly if the gas is accumulated until disk instabilities trigger star formation in regions that must necessarily be large compared to the disk extension (e.g., Noguchi 1999; Elmegreen et al. 2008, 2012a). Low metallicity and lopsidedness come together naturally in XMPs. If the physical mechanism that gives rise to the cometary shape of XMPs is also responsible for the correlation between morphology and metallicity found by Reichard et al. (2009, as an Occam's razor type of argument suggests), then triggering star formation by gas inflow must be quite common.

\subsection{Nitrogen and Oxygen in Green-pea Galaxies}

Green peas (GPs) are star-forming galaxies that receive this name because of their compactness and green color in SDSS composite images (Cardamone et al. 2009). The color is produced by an unusually large [O III] $\lambda 5007$ emission line redshifted so as to contribute to the $g$-band color. They have some of the highest specific SFRs seen in the local universe, able to double their stellar masses in a fraction of Gyr. GPs seem to be high-mass versions of the most extreme starbursting BCDs (e.g., Izotov et al. 2011; Amorín et al. 2012a) and are lowmetallicity outliers of the mass-metallicity relationship (Amorín et al. 2010, 2012a). Detailed analysis of their emission lines reveals complex kinematical structures with several components coexisting in only a few kiloparsecs, which are best interpreted as massive star-forming clumps in a dynamically young host galaxy (Amorín et al. 2012b). Even though GPs have low $\mathrm{O}$ metallicity, they present an overabundance of $\mathrm{N} / \mathrm{O}(\geqslant-1)$, which is typical of aging stellar populations. This puzzling observation is naturally explained if GPs have recently received a major flood of low-metallicity gas (Amorín et al. 2010, 2012a): the mixing with metal-poor gas reduces the metallicity (i.e., $\mathrm{O} / \mathrm{H})$, but the ratio between metal species $(\mathrm{N} / \mathrm{O})$ remains as in the original high-metallicity ISM.

Again, GPs are not special but just extreme cases in the continuous sequence of local star-forming galaxies (e.g., Izotov et al. 2011; Sánchez Almeida et al. 2013a).

\subsection{Other Hints of Gas Accretion}

The literature contains other results that are also suggestive of star formation triggered by gas accretion on a grand scale. Some of them are mentioned below.

The neutral gas distribution of the BCD galaxies often shows large distortions, with plumes and tails, and other evidence of gas inflow or outflow (e.g., Brinks \& Klein 1988; Wilcots \& Miller 1998; Lelli et al. 2012; López-Sánchez et al. 2012; Ashley et al. 2013). Such complex H i morphology appears even in the case of isolated galaxies without obvious companions (Ekta \& Chengalur 2010). The distorted gas around BCDs has all the signs of having extremely low metallicity, uncontaminated by the ongoing star formation process (e.g., Lebouteiller et al. 2013; Filho et al. 2013), which suggests that the gas is arriving rather than being expelled from the galaxy.

Even large nearby spirals show local metallicity inhomogeneities that deviate from the main gradient, e.g., M101 (Li et al. 2013). The existence of inhomogeneities is in conflict with theoretical expectations, which predict a virtually uniform distribution as a result of the short mixing timescales of the ISM, on the order of only 100 Myr (e.g., Roy \& Kunth 1995; Tenorio-Tagle 1996; de Avillez \& Mac Low 2002). Localized 
infall of metal-poor gas may be a viable alternative that explains this particular observation.

As we pointed out in connection with BCDs and GPs (Sections 5.2 and 5.4), the gas inflow produces large excursions of a galaxy in the $\mathrm{N} / \mathrm{O}$ versus $\mathrm{O} / \mathrm{H}$ plane. Numerical models by Köppen \& Hensler (2005) allow us to explain the observed distribution in irregular and spiral galaxies, but in order to reach the required large excursions, the mass of the infall gas must be much larger than the mass of the gas present in the galaxy, with the infall rate exceeding the SFR.

This trend for the low-metallicity galaxies to show anomalous metallicity gradients (Section 3) is also observed at high redshift (e.g., the $z=1.2$ MASSIV galaxies; Queyrel et al. 2012) and in several low-redshift targets (e.g., Levesque et al. 2011; Werk et al. 2010). The metal-poor galaxies tend to show a positive gradient, whereas metal-rich ones tend to show the negative one expected from secular evolution. Positive gradients naturally arise even from underlying negative gradients when metal-poor gas reaches the central regions of the disks.

\section{DISCUSSION AND CONCLUSIONS}

We measure the oxygen metallicity along the major axis of seven star-forming dwarf galaxies using different methods, including the direct method (Section 2.1 and Table 1). Two of them, J1647+21 and $\mathrm{J} 2238+14$, show drops of metallicity $(\simeq 0.5 \mathrm{dex})$ associated with enhanced star formation activity in central regions. Disk galaxies usually present a negative gradient, with the metallicity decreasing inside out. Therefore, a deficit of metallicity in the inner galaxy is strange and is attributed to the recent arrival of external metal-poor gas that has not yet mixed with the preexisting ISM (Section 1; other alternatives are also examined in Section 4). For this to happen, the incoming gas has to arrive in localized clumps rather than as an isotropic galaxy-wide accretion event. This is the explanation we suggest for the metallicity and $\mathrm{H} \alpha$ variations observed in $\mathrm{J} 2238+14$ (Figure 4) and $\mathrm{J} 1647+21$ as well (Figure 5). The image of the latter, however, may also suggest a merger event, with the main starburst at the collision point (see Figure 5, with the two colliding disks seen edge on, forming a $V$ shape in a contrived but not impossible geometry). One of the galaxies would have to be metal-poor and gas-rich, with its gas feeding the low-metallicity starburst. Actually, such a gas-rich minor merger can also be regarded as a cold-flow accretion event where the accreted gas stream is forming stars along the way (see Dekel et al. 2009a). The different morphology of J2238+14 and $\mathrm{J} 1647+21$ may be due to differences in spatial resolution, so that we have a coarser view of the former. However, we cannot discard that they reflect qualitative differences in the physical process responsible for the metallicity drops.

Our interpretation of the metallicity drops of J1647+21 and J2238+14 agrees with that given by Sánchez Almeida et al. (2013b) to explain the behavior observed in a number of local tadpole galaxies. Such agreement has several implications. It proves that galaxies other than the sample of tadpole galaxies (Miyauchi-Isobe et al. 2010; Elmegreen et al. 2012b) present the same unusual spatial metallicity pattern. The metallicity inhomogeneities of our targets were inferred using the direct method, which discards the systematic errors usually attributed to strong-line methods (e.g., Shi et al. 2005; Pérez-Montero \& Contini 2009). This source of error is discarded for $\mathrm{J} 1647+21$ and $\mathbf{J} 2238+14$, thus supporting the type of metallicity pattern disclosed in tadpoles (Sánchez Almeida et al. 2013b). Finally, the targets showing the drops tend to have a minimum metallicity smaller than one-tenth of the solar value. Something similar happens with the tadpoles analyzed by Sánchez Almeida et al. (2013b), which may suggest one-tenth of the solar metallicity to be an observational threshold for the metallicity drops to clearly show up. The origin of the threshold is unclear, but it may reveal an observational bias reflecting the degree of mixing of the galaxy gas. Assume that all typical disks have similar fairly high gas metallicity and they receive a parcel of metal-poor gas. Those galaxies that mix this metal-poor gas with the preexisting ISM before starbursting will appear as metal-rich targets of homogeneous metallicity. On the contrary, those that produce stars before mixing will look like metalpoor galaxies in integrated light, presenting large metallicity inhomogeneities. The actual threshold is probably not universal since high-redshift galaxies with drops have metallicities above the one-tenth line (Cresci et al. 2010).

If external metal-poor gas accretion feeds and triggers star formation, one would expect some kind of kinematical differences between the star-forming clumps and the underlying galaxy disk. These kinematical disturbances are predicted in numerical simulations of minor mergers and cold-flow accretion (e.g., Immeli et al. 2004; Dekel et al. 2009b; Ceverino et al. 2010) and should be sought in real galaxies. Some of them may have been observed already as, e.g., the counterrotating head found in one of the tadpole galaxies analyzed by Sánchez Almeida et al. (2013b).

Large metallicity inhomogeneities evidence a star formation driven, or at least stimulated, by pristine gas accretion. Even though the number of local galaxies showing inner metallicity inhomogeneities is still limited, there are a number of indirect hints suggesting that metal-poor gas accretion may be more than just a vestige of the early universe. The argument relies on the existence of general rules or laws followed by large numbers of galaxies that are naturally explained as star formation triggered by recent pristine gas infall. It is not the only explanation, but the inflow of pristine gas provides a simple unifying physical mechanism that explains all of them. This evidence is outlined in Section 5: among other things, the star formation dependence of the metallicity (Section 5.1), the star formation dependence of the morphology (Section 5.3), the high metallicity of quiescent BCDs (Section 5.2), and the high $\mathrm{N}$ to $\mathrm{O}$ ratio in GP galaxies (Section 5.4).

We thank R. Amorín and E. Pérez-Montero for enlightening discussions on the constraints on galaxy evolution provided by the observable N/O, V. Luridiana and M. Koleva for clarifying some of the biases of the direct method, and J. A. L. Aguerri for technical support during the observing campaigns. We also thank an anonymous referee for helping us sharpen some of the arguments in this paper. This work has been partly funded by the Spanish Ministry for Science, project AYA 2010-21887-C0404. J.M.A. acknowledges support from the European Research Council Starting Grant (SEDmorph; P.I. V. Wild). This article is based on observations made with the telescope WHT operated at the Spanish Observatorio del Roque de los Muchachos of the Instituto de Astrofísica de Canarias.

\section{REFERENCES}

Ahn, C. P., Alexandroff, R., Allende Prieto, C., et al. 2012, ApJS, 203, 21

Amorín, R., Aguerri, J. A. L., Muñoz-Tuñón, C., \& Cairós, L. M. 2009, A\&A, 501, 75

Amorín, R., Pérez-Montero, E., Vílchez, J. M., \& Papaderos, P. 2012a, ApJ, 749,185 
Amorín, R., Vílchez, J. M., Hägele, G. F., et al. 2012b, ApJL, 754, L22 Amorín, R. O., Muñoz-Tuñón, C., Aguerri, J. A. L., Cairós, L. M., \& Caon, N. 2007, A\&A, 467, 541

Amorín, R. O., Pérez-Montero, E., \& Vílchez, J. M. 2010, ApJL, 715, L128

Andrews, B. H., \& Martini, P. 2013, ApJ, 765, 140

Ashley, T., Simpson, C. E., \& Elmegreen, B. G. 2013, AJ, 146, 42

Asplund, M., Grevesse, N., Sauval, A. J., \& Scott, P. 2009, ARA\&A, 47, 481

Bell, E. F., \& de Jong, R. S. 2001, ApJ, 550, 212

Brinks, E., \& Klein, U. 1988, MNRAS, 231, 63P

Brisbin, D., \& Harwit, M. 2012, ApJ, 750, 142

Brook, C. B., Stinson, G. S., Gibson, B. K., et al. 2012, MNRAS, 426, 690

Cairós, L. M., Caon, N., Papaderos, P., et al. 2003, ApJ, 593, 312

Cardamone, C., Schawinski, K., Sarzi, M., et al. 2009, MNRAS, 399, 1191

Ceverino, D., Dekel, A., \& Bournaud, F. 2010, MNRAS, 404, 2151

Corbin, M. R., Vacca, W. D., Cid Fernandes, R., et al. 2006, ApJ, 651, 861

Cresci, G., Mannucci, F., Maiolino, R., et al. 2010, Natur, 467, 811

Davé, R., Finlator, K., \& Oppenheimer, B. D. 2012, MNRAS, 421, 98

Dayal, P., Ferrara, A., \& Dunlop, J. S. 2013, MNRAS, 430, 2891

de Avillez, M. A., \& Mac Low, M.-M. 2002, ApJ, 581, 1047

Dekel, A., \& Birnboim, Y. 2006, MNRAS, 368, 2

Dekel, A., Birnboim, Y., Engel, G., et al. 2009a, Natur, 457, 451

Dekel, A., Sari, R., \& Ceverino, D. 2009b, ApJ, 703, 785

Denicoló, G., Terlevich, R., \& Terlevich, E. 2002, MNRAS, 330, 69

Edmunds, M. G. 1990, MNRAS, 246, 678

Ekta, B., \& Chengalur, J. N. 2010, MNRAS, 403, 295

Ellison, S. L., Patton, D. R., Simard, L., \& McConnachie, A. W. 2008, ApJL, 672, L107

Elmegreen, B. G., Bournaud, F., \& Elmegreen, D. M. 2008, ApJ, 688, 67

Elmegreen, B. G., \& Elmegreen, D. M. 2010, ApJ, 722, 1895

Elmegreen, B. G., Zhang, H.-X., \& Hunter, D. A. 2012a, ApJ, 747, 105

Elmegreen, D. M., Elmegreen, B. G., Ravindranath, S., \& Coe, D. A. 2007, ApJ, 658,763

Elmegreen, D. M., Elmegreen, B. G., Sánchez Almeida, J., et al. 2012b, ApJ, 750,95

Esteban, C., Bresolin, F., Peimbert, M., et al. 2009, ApJ, 700, 654

Filho, M. E., Winkel, B., Sánchez Almeida, J., et al. 2013, A\&A, 558, A18

Gallazzi, A., Charlot, S., Brinchmann, J., White, S. D. M., \& Tremonti, C. A. 2005, MNRAS, 362, 41

Genel, S., Naab, T., Genzel, R., et al. 2012, ApJ, 745, 11

Genzel, R., Burkert, A., Bouché, N., et al. 2008, ApJ, 687, 59

Hägele, G. F., Díaz, Á. I., Terlevich, E., et al. 2008, MNRAS, 383, 209

Hidalgo, S. L., Aparicio, A., Skillman, E., et al. 2011, ApJ, 730, 14

Immeli, A., Samland, M., Westera, P., \& Gerhard, O. 2004, ApJ, 611, 20

Izotov, Y. I., Guseva, N. G., \& Thuan, T. X. 2011, ApJ, 728, 161

Kennicutt, R. C., Jr. 1998, ARA\&A, 36, 189

Kingdon, J. B., \& Ferland, G. J. 1998, ApJ, 506, 323

Kingsburgh, R. L., \& Barlow, M. J. 1994, MNRAS, 271, 257

Koleva, M., Bouchard, A., Prugniel, P., De Rijcke, S., \& Vauglin, I. 2013, MNRAS, 428, 2949

Köppen, J., \& Hensler, G. 2005, A\&A, 434, 531

Kunth, D., \& Östlin, G. 2000, A\&ARv, 10, 1

Lara-López, M. A., Cepa, J., Bongiovanni, A., et al. 2010, A\&A, 521, L53

Lebouteiller, V., Heap, S., Hubeny, I., \& Kunth, D. 2013, A\&A, 553, A16

Lee, H., Bell, E. F., \& Somerville, R. S. 2008, in IAU Symp. 255, LowMetallicity Star Formation: From the First Stars to Dwarf Galaxies, ed. L. K. Hunt, S. C. Madden, \& R. Schneider (Cambridge: Cambridge Univ. Press), 100

Lelli, F., Verheijen, M., Fraternali, F., \& Sancisi, R. 2012, A\&A, 537, A72

Levesque, E. M., Berger, E., Soderberg, A. M., \& Chornock, R. 2011, ApJ, 739,23

Li, Y., Bresolin, F., \& Kennicutt, R. C., Jr. 2013, ApJ, 766, 17

López-Sánchez, Á. R., Koribalski, B. S., van Eymeren, J., et al. 2012, MNRAS, 419, 1051
Mac Low, M.-M., \& Ferrara, A. 1999, ApJ, 513, 142

Mannucci, F., Cresci, G., Maiolino, R., Marconi, A., \& Gnerucci, A. 2010, MNRAS, 408, 2115

McGaugh, S. S. 1991, ApJ, 380, 140

Miyauchi-Isobe, N., Maehara, H., \& Nakajima, K. 2010, PNAOJ, 13, 9

Morales-Luis, A. B., Sánchez Almeida, J., Aguerri, J. A. L., \& Muñoz-Tuñón, C. 2011, ApJ, 743, 77

Moran, S. M., Heckman, T. M., Kauffmann, G., et al. 2012, ApJ, 745, 66

Noguchi, M. 1999, ApJ, 514, 77

Papaderos, P., Guseva, N. G., Izotov, Y. I., \& Fricke, K. J. 2008, A\&A, 491, 113

Papaderos, P., Loose, H.-H., Thuan, T. X., \& Fricke, K. J. 1996, A\&AS, 120, 207

Peimbert, M. 1967, ApJ, 150, 825

Peimbert, M., \& Costero, R. 1969, BOTT, 5, 3

Pérez-Montero, E., \& Contini, T. 2009, MNRAS, 398, 949

Pérez-Montero, E., Contini, T., Lamareille, F., et al. 2013, A\&A, 549, A25

Pérez-Montero, E., \& Díaz, A. I. 2003, MNRAS, 346, 105

Pettini, M., \& Pagel, B. E. J. 2004, MNRAS, 348, L59

Queyrel, J., Contini, T., Kissler-Patig, M., et al. 2012, A\&A, 539, A93

Reichard, T. A., Heckman, T. M., Rudnick, G., et al. 2009, ApJ, 691, 1005

Roy, J.-R., \& Kunth, D. 1995, A\&A, 294, 432

Sánchez Almeida, J., Aguerri, J. A. L., \& Muñoz-Tuñón, C. 2013a, RMxAA, 42, 111

Sánchez Almeida, J., Aguerri, J. A. L., Muñoz-Tuñón, C., \& Vazdekis, A. 2009, ApJ, 698, 1497

Sánchez Almeida, J., Muñoz-Tuñón, C., Amorín, R., et al. 2008, ApJ, 685, 194

Sánchez Almeida, J., Muñoz-Tuñón, C., Elmegreen, D. M., Elmegreen, B. G., \& Méndez-Abreu, J. 2013b, ApJ, 767, 74

Sánchez Almeida, J., Terlevich, R., Terlevich, E., Cid Fernandes, R., \& MoralesLuis, A. B. 2012, ApJ, 756, 163

Shaw, R. A., \& Dufour, R. J. 1995, PASP, 107, 896

Shi, F., Kong, X., Li, C., \& Cheng, F. Z. 2005, A\&A, 437, 849

Silk, J., \& Mamon, G. A. 2012, RAA, 12, 917

Skillman, E. D., Kennicutt, R. C., \& Hodge, P. W. 1989, ApJ, 347, 875

Stasińska, G. 2004, in Cosmochemistry. The Melting Pot of the Elements, ed. C. Esteban, R. García López, A. Herrero, \& F. Sánchez (Cambridge: Cambridge Univ. Press), 115

Stasińska, G. 2010, in IAU Symp. 262, Stellar Populations-Planning for the Next Decade, ed. G. R. Bruzual \& S. Charlot (Cambridge: Cambridge Univ. Press), 93

Stasińska, G., Morisset, C., Simón-Díaz, S., et al. 2013, A\&A, 551, A82

Stasińska, G., Prantzos, N., Meynet, G., et al. 2012, in EAS Publications Series, Vol. 54, Oxygen in the Universe, ed. G. Stasińska, N. Prantzos, G. Meynet, S Simón-Díaz, C. Chiappini, M. Dessauges-Zavadsky, C. Charbonnel, H.-G. Ludwig, C. Mendoza, N. Grevesse, M. Arnould, B. Barbuy, Y. Lebreton, A. Decourchelle, V. Hill, P. Ferrando, G. Hébrard, F. Durret, M. Katsuma, \& C. J. Zeippen (Les Ulis, France: EDP), 3

Stasińska, G., \& Szczerba, R. 2001, A\&A, 379, 1024

Stoughton, C., Lupton, R. H., Bernardi, M., et al. 2002, AJ, 123, 485

Straughn, A. N., Cohen, S. H., Ryan, R. E., et al. 2006, ApJ, 639, 724

Tenorio-Tagle, G. 1996, AJ, 111, 1641

Torres-Peimbert, S., \& Peimbert, M. 2003, in IAU Symp. 209, Planetary Nebulae: Their Evolution and Role in the Universe, ed. S. Kwok, M. Dopita, \& R. Sutherland (Cambridge: Cambridge Univ. Press), 363

Tremonti, C. A., Heckman, T. M., Kauffmann, G., et al. 2004, ApJ, 613, 898 van der Kruit, P. C., \& Freeman, K. C. 2011, ARA\&A, 49, 301

Vilchez, J. M., Pagel, B. E. J., Diaz, A. I., Terlevich, E., \& Edmunds, M. G. 1988, MNRAS, 235, 633

Werk, J. K., Putman, M. E., Meurer, G. R., et al. 2010, ApJ, 715, 656

Wilcots, E. M., \& Miller, B. W. 1998, AJ, 116, 2363

Windhorst, R. A., Cohen, S. H., Straughn, A. N., et al. 2006, NewAR, 50, 821

Yates, R. M., Kauffmann, G., \& Guo, Q. 2012, MNRAS, 422, 215

Zahid, H. J., Kashino, D., Silverman, J. D., et al. 2013, arXiv:1310.4950 